\title{
ARTICLE
}

\section{An AHP Approach To Develop Reference Organisms For Radiological Protection}

\author{
Zhi ZENG ${ }^{1,2,3^{*}}$, Xiujuan WANG ${ }^{1,2,3}$, Ying ZHAO ${ }^{1,2,3}$, Senming TANG $^{4}$ \\ ${ }^{I}$ Department of Engineering Physics, Tsinghua University, Beijing 100084, China \\ ${ }^{2}$ Key Laboratory of Particle \& Radiation Imaging (Tsinghua University), Ministry of Education, Beijing, 100084, China \\ ${ }^{3}$ Key Laboratory of High Energy Radiation Imaging Fundamental Science for National Defense, Beijing 100084, China \\ ${ }^{4}$ Third institute of oceanography state oceanic administration, Xiamen 361005, China
}

\begin{abstract}
It is very important to develop a set of reference organisms(ROs) for achieving radiological protection in the framework for assessing the impact of ionizing radiation on non-human species. In order to make the process of the selection of ROs more transparent and help to come to a compromise for the decision makers, a semi-quantitative model based on the fuzzy comprehensive evaluation and the AHP method to define the ROs has been established. In this work, 16 species in EPIC project are selected as an example and the concern degrees of species are calculated. It was shown that the planktotrophic mamals and carnivorous mamals have the biggest degree.
\end{abstract}

KEYWORDS: reference organisms, MCDM, fuzzy comprehensive evaluation, AHP, semi-quantitative selection

\section{Introduction}

Developing a framework for assessing the impact of ionizing radiation on non-human species is very important and ICRP had suggested that a set of quantities and units, a set of reference dose model, reference dose-per unit-intake data and reference organisms(ROs) should be developed to achieve radiological protection 1 ). As the first step, the reference organisms should be decided and then the assessment of the impact of ionizing radiation to the non-human species could be processing based on the selection fauna and flora. As a result, some international organizations and projects, such as ICRP ${ }^{1)}, \mathrm{EPIC}^{2)}, \mathrm{FASSET}^{3)}$, ERICA $^{4)}$, GRADED $^{5)}$ and IAEA ${ }^{6)}$ had 'selected' or 'suggested' their set of reference fauna and flora based on their pricinple and criteria. Compared the selection process with the different projects or organizations, the following conclusion can be drawn:

a) Qualitative and/or subjective selection. The princeple and criteria to select the ROs in different projects are descriptive and nonquantitative. It depended on the knowledge and judgment of the specialists, especially depended on the ordering of the considerable impact factors.

b) Similar criteria and shared selection strategy. The criteria of different projects are similar, including ecological status, radioecological sensitivity, radiobiological sensitivity, amenability for sampling and monitoring etc. and the strategy of selection are also similar, such as investigation of ecosystem, particular exposure pathway, and so on.

c) Incompatible and conflicting sometimes. For example, benthic bacteria are very widely distributed and exposed to the radiation, they are a potential reference organism in EPIC project $^{2)}$ while ICRP hasn't recommend them in the publication ${ }^{1)}$.

d) A mulit-criteria descision making(MCDM) process for the selection of ROs. MCDM aims at highlighting the conflicts in making numerous and conflicting evaluations and

*Corresponding Author, E-mail: zengzhi@mail.tsinghua.edu.cn deriving a way to come to a compromise in a transparent process for the decision makers.

ICRP had pointed out that a simple method of reference organisms selection should be established to reduce the conflict and be more compatible ${ }^{1)}$. In a MCDM process, fuzzy comprehensive evaluation introduced by $\mathrm{Zadeh}^{7,8)}$ and the analytic hierarchy process(AHP) introduced by Saaty ${ }^{9,10)}$ have been widely used ${ }^{11,12}$. Here we use AHP method to decide the priority of different organisms. The EPIC project will be used as an benchmark to show the whole procedure.

\section{Methods and procedure}

Step 1. Defining a set of alternative objectives.

At the beginning an investigation of the existing ecosystems in the region of interest(ROIs) is necessary. some biota and flora are suggested as the candidates of ROs after the investigation and make up a set of alternative objectives.

There are 17 species group investigated by EPIC project include benthic bacteria, macroalgae, aquatic plants, phytoplankton, zooplankton, crustaceans, molluscs, polychaetes, insect larvae, pelagic planktotrophic fish, benthic fish, pelagic carnivorous fish, amphibians, planktotrophic mamals, carnivorous mammal, benthos eating birds and fish eating birds. Benthic bacteria don't be considered for few radioecology data. The other 16 species group are chosed as a set of alternative objectives of ROs and assigned to a number from 1 to 16 in sequence. Let $X=\left\{x_{1}\right.$, $\left.x_{2}, \ldots, x_{16}\right\}$ present the alternative objectives.

\section{Step 2. Constructing the set of evaluation $\boldsymbol{V}$.}

In some extent, each species is the potential ROs to evalute the impact of ionising radiation on non-human species in ROI. Let $\mathrm{v}$ present the "degree of concern", when $v=1$ means the species is the best for ROs, otherwise $v=0$. Every species selected in EPIC project has a value between 0 and 1 and they would composed a set of $V=\left\{v_{1}, v_{2}, \ldots, v_{16}\right\}$. 


\section{Step 3. Defining the selection index set.}

There are different selecting criteria suggested by ICRP and others. To simplied the situation, 4 indexes are just used in this work:

a) Concentration ability index $u_{1}$;

b) Exposure path index $u_{2}$;

c) Radiosensitivity $u_{3}$.

d) Monitor facility and research potentiality $u_{4}$.

The concentration ability index $u_{1}$ can be presented by concentration factor $(\mathrm{CF})$. The second index $u_{2}$ includes internal and external exposure with different nuclides, and thus 4 subindexes $u_{21}, u_{22}, u_{23}$ and $u_{24}$ are used to present the $\alpha$ interal, low-energy $\beta$ interal, $\beta$ and $\gamma$ internal and $\beta$ and $\gamma$ external exposure, respectively. The third $u_{3}$ can be induced simply by half lethal dose data. And the last one $u_{4}$ depends on the pratical experience and requirentment, and can be quantified by expert system. Let $U$ be the index set, thus

$$
\begin{aligned}
U & =\left[\begin{array}{llllll}
u_{1} & u_{2} & u_{3} & u_{4}
\end{array}\right] \\
& =\left[\begin{array}{lllllll}
u_{1} & u_{21} & u_{22} & u_{23} & u_{24} & u_{3} & u_{4}
\end{array}\right],
\end{aligned}
$$

\section{Step 4. Initialing and calculate the selection index set.}

There are two methods to initial the selection index. If there are sufficient scientific and technical data, the index would be initialized directly by the data. Otherwise, the Analytic Hierarchy Process (AHP) would be applied to quantify the index.

1) Initialize concentration ability and calculate the index $u_{1}$

Concentration factor $(\mathrm{CF})$ is presented for concentration ability. The CFs of 16 speciecs group of 31 radionuclides can be derived from ERICA database. Let $C F_{\mathrm{i}, \mathrm{j}}$ present the concentration factor of the $\mathrm{i}^{\text {th }}$ species group of the $\mathrm{j}^{\text {th }}$ radionuclide, then define $p_{\mathrm{i}}$ as:

$$
p_{i}=\frac{\sum_{j=1}^{31} \frac{C F_{i, j}}{\sum_{i=1}^{16} C F_{i, j}}}{\sum_{i=1}^{16} \sum_{j=1}^{31} \frac{C F_{i, j}}{\sum_{i=1}^{16} C F_{i, j}}}
$$

And the membership function is defined as:

$$
r_{1, i}\left(p_{i}\right)=\left\{\begin{array}{cc}
0 & p_{i}<0.015 \\
\frac{p_{i}-0.015}{0.15-0.015} & 0.015 \leq p_{i}<0.15 \\
1 & p_{i} \geq 0.15
\end{array}\right.
$$

The fuzzy value $r_{1, \mathrm{i}}$ of selection index set $u_{1}$ has been got through the process. For the EPIC project, after the calculation, the fuzzy value $r_{1}$ of $u_{1}$ is $\{0.29,0.64,1.97$, $0.61,0.21,0.42,0.26,0.54,0.05,0.05,0.05,0.31,0.08$, $0.08,0.05,0.05\}$.

2) Initialize exposure path and calculate the index $u_{2}$

According to the exposure path definition of ERICA database, here 4 exposure siutation of $\alpha$ interal, low-energy $\beta$ interal, $\beta$ and $\gamma$ internal and $\beta$ and $\gamma$ external exposure are considered. And the dose convert factor(DCF) of different exposure path can be derived from ERICA database respectively. There are 63 type isotopic radionuclide include ${ }^{110 \mathrm{~m}} \mathrm{Ag},{ }^{241} \mathrm{Am},{ }^{134} \mathrm{Cs},{ }^{135} \mathrm{Cs},{ }^{136} \mathrm{Cs},{ }^{137} \mathrm{Cs}$ etc are considered in this work.

a) Internal exposure

The dose from alpha internal expsure of the $i^{\text {th }}$ species, $D_{i}^{\alpha}$ can be present by

$$
D_{i}^{\alpha}=\sum_{j=1}^{63} C_{j} \times C F_{i, j} \times D C F_{i, j}^{\alpha},
$$

where $C_{j}$ is the activity concentration in the marine, and $D C F_{i, j}^{\alpha}$ is the $\alpha$ exposure dose conversation factor of the $i^{\text {th }}$ species of $j^{\text {th }}$ isotopic radionuclides. In this work, all the $C_{j}$ are the same to $1 \mathrm{~Bq} / \mathrm{m}^{3}$.

After calculation of $D_{i}^{\alpha}$ of all 16 species finished, a pairwise comparison matrix $P$ is constructed as the following:

$$
P_{i, j}=\frac{D_{i}^{\alpha}}{D_{j}^{\alpha}},
$$

The eigenvalue of maximum of matrix $P, \lambda_{\max }$ and the largest eigenvector respectively can be calculated by mathematical tool, such as Matlab. And then, the largest eigenvector is normalized to be the initial value of $\alpha$ exposure path, denoted by $X_{i}^{\alpha}$.

The membership function of $\alpha$ internal exposure is defined like:

$$
r_{21, i}\left(x_{i}^{\alpha}\right)=\left\{\begin{array}{cc}
0 & x_{i}^{\alpha}<0.015 \\
\frac{x_{i}^{\alpha}-0.015}{0.15-0.015} & 0.015 \leq x_{i}^{\alpha}<0.15, \\
1 & x_{i}^{\alpha} \geq 0.15
\end{array}\right.
$$

After this calculation, the fuzzy value $r_{21, i}$ of $u_{21}$ is completed.

Like the above, the fuzzy value $r_{22, \mathrm{i}}$ and $r_{23, \mathrm{i}}$ of $u_{22}$ and $u_{23}$ are completed with different membership function.

b) external exposure

The dose of external exposure of $\beta$ and $\gamma$ is calculated by

$$
D_{i}^{\beta \gamma-e x}=\sum_{j=1}^{63} C_{j} \times D C F_{i, j}^{\beta \gamma-e x} \text {, }
$$

$D C F_{i, j}^{\beta \gamma-e x}$ is also the dose conversation factor derived from ERICA database. The process is like $C F$, define $q_{i}$ as

$$
q_{i}=\frac{\sum_{j=1}^{63} \frac{D C F_{i, j}}{\sum_{i=1}^{16} D C F_{i, j}}}{\sum_{i=1}^{16} \sum_{j=1}^{63} \frac{D C F_{i, j}}{\sum_{i=1}^{16} D C F_{i, j}}},
$$

And the membership function is defined by

$$
r_{24, i}\left(q_{i}\right)=\left\{\begin{array}{cc}
0 & q_{i}<0.01 \\
\frac{q_{i}-0.01}{0.1-0.01} & 0.01 \leq q_{i}<0.1, \\
1 & q_{i} \geq 0.1
\end{array}\right.
$$

The fuzzy value $r_{24, \mathrm{i}}$ of selection index set $u_{24}$ has been got through the process. 
3) Initialize radiosensitivity and calculate the index.

According to the half lethal dose data of the 16 species published by UNSCEAR ${ }^{13)}$, "point value" can be assigned by some experts and then normalized as initial value $t_{\mathrm{i}}$ for every species. The membership function can be like as:

$$
r_{3, i}\left(t_{i}\right)=\left\{\begin{array}{cc}
0 & t_{i}<0.01 \\
\frac{t_{i}-0.01}{0.1-0.01} & 0.01 \leq t_{i}<0.1, \\
1 & t_{i} \geq 0.1
\end{array}\right.
$$

4) Initialize monitor facility and research potentiality $u_{4}$

In this part, experts system is necessary for the decision process. Here a same value is appointed to every species and then normalized as initial value $s_{\mathrm{i}}$ in EPIC project. And then membeship function is defined as:

$$
r_{4, i}\left(s_{i}\right)=\left\{\begin{array}{cc}
0 & s_{i}<0.01 \\
\frac{s_{i}-0.01}{0.08-0.01} & 0.01 \leq s_{i}<0.08, \\
1 & s_{i} \geq 0.8
\end{array}\right.
$$

\section{Step 5. Calculate the weighting factor}

A pairwise comparison matrix $I$ should be decided by expert system to calculate the weighting factor of different select index and is defined as:

$$
I=\left[\begin{array}{cccc}
1 & u_{1} / u_{2} & u_{1} / u_{3} & u_{1} / u_{4} \\
u_{2} / u_{1} & 1 & u_{2} / u_{3} & u_{2} / u_{4} \\
u_{3} / u_{1} & u_{3} / u_{2} & 1 & u_{3} / u_{4} \\
u_{4} / u_{1} & u_{4} / u_{1} & u_{4} / u_{3} & 1
\end{array}\right]
$$

Integer number from 1 to 9 is used to evaluate the relative importance $u_{\mathrm{i}} / u_{\mathrm{j}}$ in $I$. The number of $u_{\mathrm{i}} / u_{\mathrm{j}}$ is more large, the relative importance of $u_{\mathrm{i}}$ compare to $u_{\mathrm{j}}$ is more important. In this work, matrix for EPIC project is assigned as:

$$
I=\left[\begin{array}{cccc}
1 & 3 / 2 & 1 / 2 & 3 \\
2 / 3 & 1 & 1 / 3 & 2 \\
2 & 3 & 1 & 4 \\
1 / 3 & 1 / 2 & 1 / 4 & 1
\end{array}\right]
$$

And then the eigenvalue of maximum of $I$ and the largest eigenvector can be calculated. After that, the largest eigenvector is normalized to be the weighting factor for different index, denoted by $W_{i}$. In EPIC project, $W=\{0.260,0.173,0.471,0.096\}$.

In the third index of exposure path criteria, there are 4 subindex discussed above. The weighting factor of different exposure can be just equivelent as the radiation weighting factors suggested by FASSET project. In particulary, the radiation weighting of $\alpha$ internal radiation is 10 , the one of low-energy $\beta$ internal radiation is 3 and the others is 1 . So the subweighting factors of $u_{21}, u_{22}, u_{23}$ and $u_{24}$ are $0.0115,0.0346$, 0.0115 and 0.0115 . So $W=\{0.260,0.0115,0.0346,0.0115$, $0.0115,0.471,0.096\}$.

\section{Step 6. Calculate the concern degree.}

All the fuzzy value $r_{\mathrm{i}}$ of different index $u_{\mathrm{i}}$ are composed into a fuzzy matrix $R$ like

$$
\begin{aligned}
R & =\left[\begin{array}{llllll}
r_{1} & r_{2} & r_{3} & r_{4}
\end{array}\right] \\
& =\left[\begin{array}{lllllll}
r_{1} & r_{21} & r_{22} & r_{23} & r_{24} & r_{3} & r_{4}
\end{array}\right],
\end{aligned}
$$

From Step 5. the weighting factor vector $W$ of EPIC project is $W=\{0.260,0.0115,0.0346,0.0115,0.0115,0.471$, 0.096 . Then the selection index matrix $U$ is:

$$
U=R \odot W
$$

Table 1, The concern degree of species in EPIC project

\begin{tabular}{|c|c|c|}
\hline No. & Species & concern degree \\
\hline $1 \#$ & Macroalgae & 0.32 \\
\hline $2 \#$ & Aquatic plants & 0.41 \\
\hline $3 \#$ & Phytoplankton & 0.46 \\
\hline $4 \#$ & Zooplankton & 0.36 \\
\hline $5 \#$ & Crustaceans & 0.39 \\
\hline $6 \#$ & Molluscs & 0.26 \\
\hline $7 \#$ & Polychaetes & 0.40 \\
\hline $8 \#$ & Insect larvae & 0.43 \\
\hline $9 \#$ & Pelagic planktotrophic fish & 0.46 \\
\hline $10 \#$ & Benthic fish & 0.46 \\
\hline $11 \#$ & Pelagic carnivorous fish & 0.46 \\
\hline $12 \#$ & Amphibians & 0.47 \\
\hline $13 \#$ & Planktotrophic mammals & 0.58 \\
\hline $14 \#$ & Carnivorous mammals & 0.58 \\
\hline $15 \#$ & Benthos eating birds & 0.54 \\
\hline $16 \#$ & Fish eating birds & 0.54 \\
\hline
\end{tabular}

In EPIC project, the result of calculation is shown in Table 1. The planktotrophic mamals and carnivorous mammals are the most important species because they have the biggest concern degree. They should be considered as one of the ROs because they are more important. On the other hand, the concern degree of molluscs is the smallest, that means it is not a favorable to choose molluscs as one of the ROs. In this way, a set of quantity of 16 species group in EPIC project is calculated by an AHP method. Because some data during the process is just decided by ourselves, the final results in this work just reflect our "selection" about EPIC project. But with the help of this mathmetical process, the final results is more legiable and directive.

\section{Conclusion}

A semi-quantitative model based on the fuzzy comprehensive evaluation and the AHP method to define the ROs in assessing the impact of ionising radiation on non-human species has been established to make a decision in definition of reference organism in a transpare process. Although the absence of some data and parameters, the method can provide a quantitative judgement to help different decision-makers to compare the set of reference organism with each other under the same criteria. 


\section{Acknowledgment}

This work was supported by NSFC Project (No. 10705019) and the scientific research foundation of MSTPRC (No. 2007050228). The author would like to express his appreciation to the comments and suggestions from the referees.

\section{References}

1) ICRP, A Framework for Assessing the Impact of Ionizing Radiation on Non-Human Species, ICRP Publication 91, International Commission on Radiological Protection, (2003).

2) N. A. Beresford, S. M. Wright, T. Sazykin, A deliverable report for EPIC: Reference arctic organisms, ICA2-CT-2000-10032, (2001).

3) Per Strand, Nick Beresford, Rodolfo Avila, Steve R. Jones, Carl-Magnus Larsson, FASSET: Identification of candidate reference organisms from a radiation exposure pathways perspective, deliverable 1, Swedish Radiation Protection Authority, (2001).

4) B. Nick, B. Justin, C. David, G-L. Jacqueline, H. Brenda, L. Carl-Magus, O. Deborah, P. Gerhard, Z. Irene, D-ERICA: An intergrated approach to the assessment and management of environmental risks from ionising radiation, FI6R-CT-2004-508847, European commission community research, (2007).
5) DOE, RESRAD-BIOTA: A tool for implementing a Graded approach to biota dose evaluation, DOE/EH-0676, United States Department of Energy, (2004).

6) IAEA, Review of the selection criteria used by different biota dose assessment models in the selection of reference organisms, International Atomic Energy Agency, (2005) .

7) R. Bellman, L.A. Zadeh, "Decision making in a fuzzy environment," Management Sci., 17,141-164(1990).

8) L.A. Zadeh, "Fuzzy sets," Inform. and Control, 8, 338-356 (1965).

9) T. L. Saaty, The Analytic Hierarchy Process, McGraw Hill, New York:. (1980).

10) T. L. Saaty, "Negative priorities in the analytic hierarchy process," Mathematical and Computer Modelling, 37, 1063-1075 (2003b).

11) Tam CM, Tong TKL, Wong YW. , "Selection of Concrete Pump Using the Superiority and Inferiority Ranking Method," Journal of Construction Engineering and Management, 130[6], 827-834 (2004).

12) Tam CM, Tong TKL, Leung AWT and Chiu GWC, "Site Layout Planning using Nonstructural Fuzzy Decision Support System," Journal of Construction Engineering and Management, 128[3], 220-231(2002)

13) UNSCEAR. Sources and effects of ionizing radiation, UNSCEAR 1996 Report, United Nations Scientific Committee on the Effects of Atomic Radiation, (1996). 